\title{
An extended Hilbert's integral inequality in the whole plane with parameters
}

Leping $\mathrm{He}^{1 *}$, Yin $\mathrm{Li}^{1}$ and Bicheng Yang ${ }^{2}$

"Correspondence:

jdheleping@163.com

'College of Mathematics and

Statistics, Jishou University, Jishou,

P.R. China

Full list of author information is

available at the end of the article

\begin{abstract}
By introducing independent parameters and interval variables, applying the weight functions and the technique of real analysis, an extended Hilbert's integral inequality in the whole plane with parameters and a best possible constant factor is provided. The equivalent forms, the reverses, and the related homogeneous forms with particular parameters are considered. Meanwhile, an extended Hilbert's integral operator in the whole plane is defined, and the operator expressions for the equivalent inequalities are obtained.
\end{abstract}

MSC: 26D15; 47A05

Keywords: Hilbert's integral inequality; Weight function; Equivalent form; Operator; Norm

\section{Introduction}

Assuming that $0<\int_{0}^{\infty} f^{2}(x) d x<\infty$ and $0<\int_{0}^{\infty} g^{2}(y) d y<\infty$, we have the following wellknown Hilbert's integral inequality with the best possible constant factor $\pi$ [1]:

$$
\int_{0}^{\infty} \int_{0}^{\infty} \frac{f(x) g(y)}{x+y} d x d y<\pi\left(\int_{0}^{\infty} f^{2}(x) d x \int_{0}^{\infty} g^{2}(y) d y\right)^{\frac{1}{2}}
$$

In 1925, Hardy gave an extension of (1) as follows [2]: If $p>1, \frac{1}{p}+\frac{1}{q}=1, f(x) \geq 0$, satisfying $0<\int_{0}^{\infty} f^{p}(x) d x<\infty$, and $g(y) \geq 0$, satisfying $0<\int_{0}^{\infty} g^{q}(y) d y<\infty$, then we have

$$
\int_{0}^{\infty} \int_{0}^{\infty} \frac{f(x) g(y)}{x+y} d x d y<\frac{\pi}{\sin \left(\frac{\pi}{p}\right)}\left(\int_{0}^{\infty} f^{p}(x) d x\right)^{\frac{1}{p}}\left(\int_{0}^{\infty} g^{q}(y) d y\right)^{\frac{1}{q}},
$$

where the constant factor $\pi / \sin \left(\frac{\pi}{p}\right)$ is still the best possible. We call (2) Hardy-Hilbert's integral inequality, which with (1) is important in analysis and its applications (cf. [1, 3]). In 1934, Hardy et al. gave an extension of (2) with the general homogeneous kernel of degree -1 (see [1], Theorem 319). Meanwhile, a Hilbert-type integral inequality with the general nonhomogeneous kernel is provided (see [1], Theorem 350): If $h(x)>0, \int_{0}^{\infty} h(x) x^{s-1} d x=$ $\phi(s) \in \mathbf{R}_{+}=(0, \infty)$, then

$$
\int_{0}^{\infty} \int_{0}^{\infty} h(x y) f(x) g(y) d x d y<\phi\left(\frac{1}{p}\right)\left(\int_{0}^{\infty} x^{p-2} f^{p}(x) d x\right)^{\frac{1}{p}}\left(\int_{0}^{\infty} g^{q}(y) d y\right)^{\frac{1}{q}} .
$$

(0) The Author(s) 2018. This article is distributed under the terms of the Creative Commons Attribution 4.0 International License (http://creativecommons.org/licenses/by/4.0/), which permits unrestricted use, distribution, and reproduction in any medium, provided you give appropriate credit to the original author(s) and the source, provide a link to the Creative Commons license, and indicate if changes were made. 
By introducing an independent parameter $\lambda \in(0, \infty)$ and the beta function, in 1998, Yang [4] gave an extension of (1) as follows:

$$
\int_{0}^{\infty} \int_{0}^{\infty} \frac{f(x) g(y)}{(x+y)^{\lambda}} d x d y<B\left(\frac{\lambda}{2}, \frac{\lambda}{2}\right)\left(\int_{0}^{\infty} x^{1-\lambda} f^{2}(x) d x \int_{0}^{\infty} y^{1-\lambda} g^{2}(y) d y\right)^{\frac{1}{2}}
$$

where the constant factor $B\left(\frac{\lambda}{2}, \frac{\lambda}{2}\right)$ is the best possible, and

$$
B(u, v):=\int_{0}^{\infty} \frac{t^{v-1}}{(1+t)^{u+v}} d t \quad(u, v>0)
$$

is the beta function (cf. [5]).

In 2007, Li [6] gave an extension of (4) and Yang [7] provided the following Hilbert-type integral inequality with the nonhomogeneous kernel:

$$
\int_{0}^{\infty} \int_{0}^{\infty} \frac{f(x) g(y)}{1+(x y)^{\lambda}} d x d y<\frac{\pi}{\lambda}\left(\int_{0}^{\infty} x^{p\left(1-\frac{\lambda}{2}\right)-1} f^{p}(x) d x\right)^{\frac{1}{p}}\left(\int_{0}^{\infty} y^{q\left(1-\frac{\lambda}{2}\right)-1} g^{q}(y) d y\right)^{\frac{1}{q}} .
$$

Since then, a lot of authors have continued to discuss this topic (cf. [8-14]).

In this paper, by introducing independent parameters and interval variables, applying the weight functions and the technique of real analysis, a Hilbert-type integral inequality in the whole plane with parameters and a best possible constant factor is provided as follows:

$$
\begin{aligned}
& \int_{-\infty}^{\infty} \int_{-\infty}^{\infty} \frac{f(x) g(y)}{(|x|+|y|)^{\lambda}} d x d y \\
& \quad<2 B(\mu, \sigma)\left[\int_{-\infty}^{\infty}|x|^{p(1-\mu)-1} f^{p}(x) d x\right]^{\frac{1}{p}}\left[\int_{-\infty}^{\infty}|y|^{q(1-\sigma)-1} g^{q}(y) d y\right]^{\frac{1}{q}}
\end{aligned}
$$

$(\mu, \sigma>0, \mu+\sigma=\lambda)$, which is an extension of (4). The more general form of (6) with parameters, the equivalent inequalities, the reverses, and the related homogeneous form with the particular parameter are considered. Meanwhile, an extended Hilbert's integral operator in the whole plane is defined, and the operator expressions for the equivalent inequalities are obtained.

\section{Weight functions and an initial inequality}

Definition 1 Suppose that $\delta \in\{-1,1\},-1<\alpha, \beta<1, \mu, \sigma>0, \mu+\sigma=\lambda$. Define the following weight functions:

$$
\begin{aligned}
& \omega_{\delta}(\sigma, y):=(|y|+\beta y)^{\sigma} \int_{-\infty}^{\infty} \frac{(|x|+\alpha x)^{\delta \sigma-1}}{\left[1+(|x|+\alpha x)^{\delta}(|y|+\beta y)\right]^{\lambda}} d x \quad(y \in \mathbf{R}=(-\infty, \infty)), \\
& \varpi_{\delta}(\sigma, x):=(|x|+\alpha x)^{\delta \sigma} \int_{-\infty}^{\infty} \frac{(|y|+\beta y)^{\sigma-1}}{\left[1+(|x|+\alpha x)^{\delta}(|y|+\beta y)\right]^{\lambda}} d y \quad(x \in \mathbf{R}) .
\end{aligned}
$$


We find

$$
\begin{aligned}
& \omega_{\delta}(\sigma, y) \\
& \quad=(|y|+\beta y)^{\sigma}\left\{\int_{-\infty}^{0} \frac{(-x+\alpha x)^{\delta \sigma-1} d x}{\left[1+(-x+\alpha x)^{\delta}(|y|+\beta y)\right]^{\lambda}}+\int_{0}^{\infty} \frac{(x+\alpha x)^{\delta \sigma-1} d x}{\left[1+(x+\alpha x)^{\delta}(|y|+\beta y)\right]^{\lambda}}\right\} \\
& \quad=(|y|+\beta y)^{\sigma}\left\{\int_{0}^{\infty} \frac{(x-\alpha x)^{\delta \sigma-1} d x}{\left[1+(x-\alpha x)^{\delta}(|y|+\beta y)\right]^{\lambda}}+\int_{0}^{\infty} \frac{(x+\alpha x)^{\delta \sigma-1} d x}{\left[1+(x+\alpha x)^{\delta}(|y|+\beta y)\right]^{\lambda}}\right\}
\end{aligned}
$$

For fixed $y(\neq 0)$, setting $u=(x-\alpha x)^{\delta}(|y|+\beta y)$ in the above first integral, we obtain that $x=\frac{(|y|+\beta y)^{-\frac{1}{\delta}}}{1-\alpha} u^{\frac{1}{\delta}}, d x=\frac{(|y|+\beta y)^{-\frac{1}{\delta}}}{\delta(1-\alpha)} u^{\frac{1}{\delta}-1} d u$, and

$$
\begin{aligned}
\int_{0}^{\infty} \frac{(x-\alpha x)^{\delta \sigma-1} d x}{\left[1+(x-\alpha x)^{\delta}(|y|+\beta y)\right]^{\lambda}} & =\int_{0}^{\infty} \frac{u^{\sigma-\frac{1}{\delta}}}{(1+u)^{\lambda}(|y|+\beta y)^{\sigma-\frac{1}{\delta}}} \frac{u^{\frac{1}{\delta}-1}}{(1-\alpha)(|y|+\beta y)^{\frac{1}{\delta}}} d u \\
& =\frac{1}{(1-\alpha)(|y|+\beta y)^{\sigma}} \int_{0}^{\infty} \frac{u^{\sigma-1}}{(1+u)^{\lambda}} d u .
\end{aligned}
$$

In the same way, setting $u=(x+\alpha x)^{\delta}(|y|+\beta y)$ in the above second integral, it follows that

$$
\int_{0}^{\infty} \frac{(x+\alpha x)^{\delta \sigma-1} d x}{\left[1+(x+\alpha x)^{\delta}(|y|+\beta y)\right]^{\lambda}}=\frac{1}{(1+\alpha)(|y|+\beta y)^{\sigma}} \int_{0}^{\infty} \frac{u^{\sigma-1}}{(1+u)^{\lambda}} d u
$$

Hence, we have

$$
\omega_{\delta}(\sigma, y)=\left(\frac{1}{1-\alpha}+\frac{1}{1+\alpha}\right) \int_{0}^{\infty} \frac{u^{\sigma-1}}{(1+u)^{\lambda}} d u=K_{\alpha}(\sigma):=\frac{2}{1-\alpha^{2}} B(\mu, \sigma) .
$$

By (8), we find

$$
\begin{aligned}
& \varpi_{\delta}(\sigma, x) \\
& \quad=(|x|+\alpha x)^{\delta \sigma}\left\{\int_{-\infty}^{0} \frac{(-y+\beta y)^{\sigma-1} d y}{\left[1+(|x|+\alpha x)^{\delta}(-y+\beta y)\right]^{\lambda}}+\int_{0}^{\infty} \frac{(y+\beta y)^{\sigma-1} d y}{\left[1+(|x|+\alpha x)^{\delta}(y+\beta y)\right]^{\lambda}}\right\} \\
& \quad=(|x|+\alpha x)^{\delta \sigma}\left\{\int_{0}^{\infty} \frac{(y-\beta y)^{\sigma-1} d y}{\left[1+(|x|+\alpha x)^{\delta}(y-\beta y)\right]^{\lambda}}+\int_{0}^{\infty} \frac{(y+\beta y)^{\sigma-1} d y}{\left[1+(|x|+\alpha x)^{\delta}(y+\beta y)\right]^{\lambda}}\right\} .
\end{aligned}
$$

For fixed $x(\neq 0)$, setting $u=(|x|+\alpha x)^{\delta}(1-\beta) y$ in the above first integral, we obtain $y=$ $\frac{u}{(1-\beta)(|x|+\alpha x)^{\delta}}$, and

$$
\begin{aligned}
\int_{0}^{\infty} \frac{(y-\beta y)^{\sigma-1} d y}{\left[1+(|x|+\alpha x)^{\delta}(y-\beta y)\right]^{\lambda}} & =\int_{0}^{\infty} \frac{u^{\sigma-1}}{(1+u)^{\lambda}(|x|+\alpha x)^{\delta(\sigma-1)}} \frac{d u}{(1-\beta)(|x|+\alpha x)^{\delta}} \\
& =\frac{1}{(1-\beta)(|x|+\alpha x)^{\delta \sigma}} \int_{0}^{\infty} \frac{u^{\sigma-1}}{(1+u)^{\lambda}} d u .
\end{aligned}
$$

In the same way, setting $u=(|x|+\alpha x)^{\delta}(1+\beta) y$ in the above second integral, we find

$$
\int_{0}^{\infty} \frac{(y+\beta y)^{\sigma-1} d y}{\left[1+(|x|+\alpha x)^{\delta}(y+\beta y)\right]^{\lambda}}=\frac{1}{(1+\beta)(|x|+\alpha x)^{\delta \sigma}} \int_{0}^{\infty} \frac{u^{\sigma-1}}{(1+u)^{\lambda}} d u
$$


and then

$$
\varpi_{\delta}(\sigma, x)=\left(\frac{1}{1-\beta}+\frac{1}{1+\beta}\right) \int_{0}^{\infty} \frac{u^{\sigma-1}}{(1+u)^{\lambda}} d u=K_{\beta}(\sigma):=\frac{2}{1-\beta^{2}} B(\mu, \sigma) .
$$

Theorem 1 Suppose that $p>0(p \neq 1), \frac{1}{p}+\frac{1}{q}=1, \delta \in\{-1,1\},-1<\alpha, \beta<1, \mu, \sigma>0, \mu+\sigma=$ $\lambda$, and

$$
K(\sigma):=K_{\beta}^{\frac{1}{p}}(\sigma) K_{\alpha}^{\frac{1}{q}}(\sigma)=\frac{2 B(\mu, \sigma)}{\left(1-\beta^{2}\right)^{1 / p}\left(1-\alpha^{2}\right)^{1 / q}} .
$$

If $f(x) \geq 0(x \in \boldsymbol{R})$, satisfying $0<\int_{-\infty}^{\infty}(|x|+\alpha x)^{p(1-\delta \sigma)-1} f^{p}(x) d x<\infty$, then

(i) for $p>1$, we have the following inequality:

$$
\begin{aligned}
J & :=\left\{\int_{-\infty}^{\infty}(|y|+\beta y)^{p \sigma-1}\left[\int_{-\infty}^{\infty} \frac{f(x)}{\left[1+(|x|+\alpha x)^{\delta}(|y|+\beta y)\right]^{\lambda}} d x\right]^{p} d y\right\}^{\frac{1}{p}} \\
& <\frac{2 B(\mu, \sigma)}{\left(1-\beta^{2}\right)^{1 / p}\left(1-\alpha^{2}\right)^{1 / q}}\left[\int_{-\infty}^{\infty}(|x|+\alpha x)^{p(1-\delta \sigma)-1} f^{p}(x) d x\right]^{\frac{1}{p}}
\end{aligned}
$$

(ii) for $0<p<1$, we have the reverse of (12).

Proof (i) For $p>1$, by Hölder's inequality with weight [15] and (7), when $y \neq 0$, we find

$$
\begin{aligned}
\int_{-\infty}^{\infty} & \frac{f(x)}{\left[1+(|x|+\alpha x)^{\delta}(|y|+\beta y)\right]^{\lambda}} d x \\
= & \int_{-\infty}^{\infty} \frac{1}{\left[1+(|x|+\alpha x)^{\delta}(|y|+\beta y)\right]^{\lambda}}\left[\frac{(|x|+\alpha x)^{(1-\delta \sigma) / q}}{(|y|+\beta y)^{(1-\sigma) / p}} f(x)\right]\left[\frac{(|y|+\beta y)^{(1-\sigma) / p}}{(|x|+\alpha x)^{(1-\delta \sigma) / q}}\right] d x \\
\leq & \left\{\int_{-\infty}^{\infty} \frac{(|x|+\alpha x)^{(1-\delta \sigma)(p-1)}}{\left[1+(|x|+\alpha x)^{\delta}(|y|+\beta y)\right]^{\lambda}} \frac{f^{p}(x) d x}{(|y|+\beta y)^{1-\sigma}}\right\}^{\frac{1}{p}} \\
& \times\left\{\int_{-\infty}^{\infty} \frac{(|y|+\beta y)^{(1-\sigma)(q-1)}}{\left[1+(|x|+\alpha x)^{\delta}(|y|+\beta y)\right]^{\lambda}} \frac{d x}{(|x|+\alpha x)^{1-\delta \sigma}}\right\}^{\frac{1}{q}} \\
= & \left(\omega_{\delta}(\sigma, y)\right)^{\frac{1}{q}}(|y|+\beta y)^{\frac{1}{p}-\sigma}\left\{\int_{-\infty}^{\infty} \frac{(|x|+\alpha x)^{(1-\delta \sigma)(p-1)}}{\left[1+(|x|+\alpha x)^{\delta}(|y|+\beta y)\right]^{\lambda}} \frac{f^{p}(x) d x}{(|y|+\beta y)^{1-\sigma}}\right\}^{\frac{1}{p}} .
\end{aligned}
$$

We prove that (13) takes the form of strict inequality. Otherwise, there exists $y \neq 0$ such that (13) takes the form of equality. Then there exist constants A and B such that they are not all zero, and [15]

$$
\begin{aligned}
& A \frac{(|x|+\alpha x)^{(1-\delta \sigma)(p-1)}}{\left[1+(|x|+\alpha x)^{\delta}(|y|+\beta y)\right]^{\lambda}} \frac{f^{p}(x)}{(|y|+\beta y)^{1-\sigma}} \\
& \quad=B \frac{(|y|+\beta y)^{(1-\sigma)(q-1)}}{\left[1+(|x|+\alpha x)^{\delta}(|y|+\beta y)\right]^{\lambda}} \frac{1}{(|x|+\alpha x)^{1-\delta \sigma}} \quad \text { a.e. in } \mathbf{R} .
\end{aligned}
$$

If $A=0$, then $B=0$, which is impossible. We suppose that $A \neq 0$, namely

$$
(|x|+\alpha x)^{p(1-\delta \sigma)-1} f^{p}(x)=\frac{B(|y|+\beta y)^{q(1-\sigma)}}{A(|x|+\alpha x)} \text { a.e. in } \mathbf{R},
$$

which contradicts the fact that $0<\int_{-\infty}^{\infty}(|x|+\alpha x)^{p(1-\delta \sigma)-1} f^{p}(x) d x<\infty$. 
Then by (11) and Fubini's theorem [16], we find

$$
\begin{aligned}
J & <K_{\alpha}^{\frac{1}{q}}(\sigma)\left\{\int_{-\infty}^{\infty} \int_{-\infty}^{\infty} \frac{(|x|+\alpha x)^{(1-\delta \sigma)(p-1)}}{\left[1+(|x|+\alpha x)^{\delta}(|y|+\beta y)\right]^{\lambda}} \frac{1}{(|y|+\beta y)^{1-\sigma}} f^{p}(x) d x d y\right\}^{\frac{1}{p}} \\
& =K_{\alpha}^{\frac{1}{q}}(\sigma)\left\{\int_{-\infty}^{\infty} \varpi_{\delta}(\sigma, x)(|x|+\alpha x)^{p(1-\delta \sigma)-1} f^{p}(x) d x\right\}^{\frac{1}{p}} .
\end{aligned}
$$

In view of (10) and (11), we have (12).

(ii) For $0<p<1$, by the reverse Hölder's inequality [15], (7), and (9), we have the reverses of (13) and (14). Then, by (10) and (11), we obtain the reverse of (12).

The theorem is proved.

\section{Main results}

Theorem 2 Suppose that $p>1, \frac{1}{p}+\frac{1}{q}=1, \delta \in\{-1,1\},-1<\alpha, \beta<1, \mu, \sigma>0, \mu+\sigma=\lambda$. If $f(x), g(y) \geq 0$, satisfying

$$
0<\int_{-\infty}^{\infty}(|x|+\alpha x)^{p(1-\delta \sigma)-1} f^{p}(x) d x<\infty \quad \text { and } \quad 0<\int_{-\infty}^{\infty}(|y|+\beta y)^{q(1-\sigma)-1} g^{q}(y) d y<\infty
$$

then we have the following inequality equivalent to (12):

$$
\begin{aligned}
I:= & \int_{-\infty}^{\infty} \int_{-\infty}^{\infty} \frac{f(x) g(y)}{\left[1+(|x|+\alpha x)^{\delta}(|y|+\beta y)\right]^{\lambda}} d x d y \\
< & \frac{2 B(\mu, \sigma)}{\left(1-\beta^{2}\right)^{1 / p}\left(1-\alpha^{2}\right)^{1 / q}}\left[\int_{-\infty}^{\infty}(|x|+\alpha x)^{p(1-\delta \sigma)-1} f^{p}(x) d x\right]^{\frac{1}{p}} \\
& \times\left[\int_{-\infty}^{\infty}(|y|+\beta y)^{q(1-\sigma)-1} g^{q}(y) d y\right]^{\frac{1}{q}}
\end{aligned}
$$

where the constant $K(\sigma)=\frac{2 B(\mu, \sigma)}{\left(1-\beta^{2}\right)^{1 / p}\left(1-\alpha^{2}\right)^{1 / q}}$ in (15) and (12) is the best possible.

In particular, for $\delta=1$, we have the following equivalent inequalities with the nonhomogeneous kernel and the best possible constant factor $K(\sigma)=\frac{2 B(\mu, \sigma)}{\left(1-\beta^{2}\right)^{1 / p}\left(1-\alpha^{2}\right)^{1 / q}}$ :

$$
\begin{aligned}
& \left\{\int_{-\infty}^{\infty}(|y|+\beta y)^{p \sigma-1}\left[\int_{-\infty}^{\infty} \frac{f(x)}{[1+(|x|+\alpha x)(|y|+\beta y)]^{\lambda}} d x\right]^{p} d y\right\}^{\frac{1}{p}} \\
& <\frac{2 B(\mu, \sigma)}{\left(1-\beta^{2}\right)^{1 / p}\left(1-\alpha^{2}\right)^{1 / q}}\left[\int_{-\infty}^{\infty}(|x|+\alpha x)^{p(1-\sigma)-1} f^{p}(x) d x\right]^{\frac{1}{p}} \\
& \int_{-\infty}^{\infty} \int_{-\infty}^{\infty} \frac{f(x) g(y)}{[1+(|x|+\alpha x)(|y|+\beta y)]^{\lambda}} d x d y \\
& <\frac{2 B(\mu, \sigma)}{\left(1-\beta^{2}\right)^{1 / p}\left(1-\alpha^{2}\right)^{1 / q}\left[\int_{-\infty}^{\infty}(|x|+\alpha x)^{p(1-\sigma)-1} f^{p}(x) d x\right]^{\frac{1}{p}}} \\
& \quad \times\left[\int_{-\infty}^{\infty}(|y|+\beta y)^{q(1-\sigma)-1} g^{q}(y) d y\right]^{\frac{1}{q}} .
\end{aligned}
$$


Proof By Hölder's inequality, we find

$$
\begin{aligned}
I & =\int_{-\infty}^{\infty}\left\{(|y|+\beta y)^{\sigma-\frac{1}{p}} \int_{-\infty}^{\infty} \frac{f(x)}{[1+(|x|+\alpha x)(|y|+\beta y)]^{\lambda}} d x\right\}\left[(|y|+\beta y)^{\frac{1}{p}-\sigma} g(y)\right] d y \\
& \leq J\left[\int_{-\infty}^{\infty}(|y|+\beta y)^{q(1-\sigma)-1} g^{q}(y) d y\right]^{\frac{1}{q}},
\end{aligned}
$$

and then by (12) we have (15).

On the other hand, suppose that (15) is valid. We set

$$
g(y):=(|y|+\beta y)^{p \sigma-1}\left\{\int_{-\infty}^{\infty} \frac{f(x)}{\left[1+(|x|+\alpha x)^{\delta}(|y|+\beta y)\right]^{\lambda}} d x\right\}^{p-1} \quad(y \in \mathbf{R}) .
$$

By (14) and the assumptions, we find $J<\infty$. If $J=0$, then (12) is trivially valid; if $J>0$, then by (15) we obtain

$$
\begin{aligned}
0< & \int_{-\infty}^{\infty}(|y|+\beta y)^{q(1-\sigma)-1} g^{q}(y) d y=J^{p}=I \\
< & K(\sigma)\left[\int_{-\infty}^{\infty}(|x|+\alpha x)^{p(1-\delta \sigma)-1} f^{p}(x) d x\right]^{\frac{1}{p}} \\
& \times\left[\int_{-\infty}^{\infty}(|y|+\beta y)^{q(1-\sigma)-1} g^{q}(y) d y\right]^{\frac{1}{q}} \\
< & \infty, \\
J= & {\left[\int_{-\infty}^{\infty}(|y|+\beta y)^{q(1-\sigma)-1} g^{q}(y) d y\right]^{\frac{1}{p}} } \\
< & K(\sigma)\left[\int_{-\infty}^{\infty}(|x|+\alpha x)^{p(1-\delta \sigma)-1} f^{p}(x) d x\right]^{\frac{1}{p}} .
\end{aligned}
$$

Hence, we have (12), which is equivalent to (15).

For $n \in \mathbf{N}=\{1,2, \ldots\}, n>\frac{1}{q \mu}$, we define the sets $E_{\delta}:=\left\{x \in \mathbf{R} ;|x|^{\delta} \geq 1\right\}$,

$$
E_{\delta}^{+}:=\left\{x \in \mathbf{R}_{+} ; x^{\delta} \geq 1\right\}, \quad E_{\delta}^{-}:=\left\{-x \in \mathbf{R}_{+} ;(-x)^{\delta} \geq 1\right\},
$$

and the following functions:

$$
\begin{aligned}
& \tilde{f}(x):= \begin{cases}(|x|+\alpha x)^{\delta\left(\sigma-\frac{1}{n p}\right)-1}, & x \in E_{\delta}, \\
0, & x \in \mathbf{R} \backslash E_{\delta},\end{cases} \\
& \tilde{g}(y):= \begin{cases}(|y|+\beta y)^{\sigma+\frac{1}{n q}-1}, & y \in[-1,1], \\
0, & y \in(-\infty,-1) \cup(1, \infty) .\end{cases}
\end{aligned}
$$


He et al. Journal of Inequalities and Applications ( 2018) 2018:216

Page 7 of 11

Then we obtain that

$$
\begin{aligned}
& \int_{E_{\delta}}(|x|+\alpha x)^{-\frac{\delta}{n}-1} d x \\
& =\int_{E_{\delta}^{-}}(-x+\alpha x)^{-\frac{\delta}{n}-1} d x+\int_{E_{\delta}^{+}}(x+\alpha x)^{-\frac{\delta}{n}-1} d x \\
& =\left[(1-\alpha)^{-\frac{\delta}{n}-1}+(1+\alpha)^{-\frac{\delta}{n}-1}\right] \int_{E_{\delta}^{+}} x^{-\frac{\delta}{n}-1} d x \\
& =\left[(1-\alpha)^{-\frac{\delta}{n}-1}+(1+\alpha)^{-\frac{\delta}{n}-1}\right] \int_{1}^{\infty}\left(u^{\delta^{-1}}\right)^{-\frac{\delta}{n}-1}\left|\delta^{-1}\right| u^{\delta^{-1}-1} d u \quad\left(u=x^{\delta}\right) \\
& =\left[(1-\alpha)^{-\frac{\delta}{n}-1}+(1+\alpha)^{-\frac{\delta}{n}-1}\right] \int_{1}^{\infty} u^{-\frac{1}{n}-1} d u=\left[(1-\alpha)^{-\frac{\delta}{n}-1}+(1+\alpha)^{-\frac{\delta}{n}-1}\right] n, \\
& \int_{-1}^{1}(|y|+\beta y)^{\frac{1}{n}-1} d y \\
& =\int_{-1}^{0}(-y+\beta y)^{\frac{1}{n}-1} d y+\int_{0}^{1}(y+\beta y)^{\frac{1}{n}-1} d y \\
& =\left[(1-\beta)^{\frac{1}{n}-1}+(1+\beta)^{\frac{1}{n}-1}\right] \int_{0}^{1} y^{\frac{1}{n}-1} d y=\left[(1-\beta)^{\frac{1}{n}-1}+(1+\beta)^{\frac{1}{n}-1}\right] n, \\
& \tilde{L}:=\left[\int_{-\infty}^{\infty}(|x|+\alpha x)^{p(1-\delta \sigma)-1} \tilde{f}^{p}(x) d x\right]^{\frac{1}{p}}\left[\int_{-\infty}^{\infty}(|y|+\beta y)^{q(1-\sigma)-1} \tilde{g}^{q}(y) d y\right]^{\frac{1}{q}} \\
& =\left[\int_{E_{\delta}}(|x|+\alpha x)^{-\frac{\delta}{n}-1} d x\right]^{\frac{1}{p}}\left[\int_{-1}^{1}(|y|+\beta y)^{\frac{1}{n}-1} d y\right]^{\frac{1}{q}} \\
& =n\left[(1-\alpha)^{-\frac{\delta}{n}-1}+(1+\alpha)^{-\frac{\delta}{n}-1}\right]^{\frac{1}{p}}\left[(1-\beta)^{\frac{1}{n}-1}+(1+\beta)^{\frac{1}{n}-1}\right]^{\frac{1}{q}} .
\end{aligned}
$$

In the same way, we still find that

$$
\begin{aligned}
& \int_{E_{\delta}}(|x|+\alpha x)^{-\delta\left(\mu+\frac{1}{n p}\right)-1} d x=\left[(1-\alpha)^{-\delta\left(\mu+\frac{1}{n p}\right)-1}+(1+\alpha)^{-\delta\left(\mu+\frac{1}{n p}\right)-1}\right] \frac{1}{\mu+\frac{1}{n p}} \\
& \int_{\mathbf{R} \backslash[-1,1]}(|y|+\beta y)^{-\left(\mu-\frac{1}{n q}\right)-1} d y=\left[(1-\beta)^{-\left(\mu-\frac{1}{n q}\right)-1}+(1+\beta)^{-\left(\mu-\frac{1}{n q}\right)-1}\right] \frac{1}{\mu-\frac{1}{n q}} .
\end{aligned}
$$

Hence, we obtain

$$
\begin{aligned}
\tilde{I}:= & \int_{-\infty}^{\infty} \int_{-\infty}^{\infty} \frac{\tilde{f}(x) \tilde{g}(y)}{\left[1+(|x|+\alpha x)^{\delta}(|y|+\beta y)\right]^{\lambda}} d y d x \\
= & \int_{E_{\delta}}\left[\int_{-1}^{1} \frac{(|x|+\alpha x)^{\delta\left(\sigma-\frac{1}{n p}\right)-1}(|y|+\beta y)^{\left(\sigma+\frac{1}{n q}\right)-1}}{\left[1+(|x|+\alpha x)^{\delta}(|y|+\beta y)\right]^{\lambda}} d y\right] d x \\
= & \int_{E_{\delta}}(|x|+\alpha x)^{\frac{-\delta}{n}-1} \varpi_{\delta}\left(\sigma+\frac{1}{n q}, x\right) d x \\
& -\int_{E_{\delta}}\left[\int_{\mathbf{R} \backslash[-1,1]} \frac{(|x|+\alpha x)^{\delta\left(\sigma-\frac{1}{n p}\right)-1}(|y|+\beta y)^{\sigma+\frac{1}{n q}-1}}{\left[1+(|x|+\alpha x)^{\delta}(|y|+\beta y)\right]^{\lambda}} d y\right] d x \\
\geq & \int_{E_{\delta}}(|x|+\alpha x)^{\frac{-\delta}{n}-1} \varpi_{\delta}\left(\sigma+\frac{1}{n q}, x\right) d x
\end{aligned}
$$




$$
\begin{aligned}
& -\int_{E_{\delta}}\left[\int_{\mathbf{R} \backslash[-1,1]} \frac{(|x|+\alpha x)^{\delta\left(\sigma-\frac{1}{n p}\right)-1}(|y|+\beta y)^{\sigma+\frac{1}{n q}-1}}{\left[(|x|+\alpha x)^{\delta}(|y|+\beta y)\right]^{\lambda}} d y\right] d x \\
= & K_{\beta}\left(\sigma+\frac{1}{n q}\right) \int_{E_{\delta}}(|x|+\alpha x)^{\frac{-\delta}{n}-1} d x \\
& -\int_{E_{\delta}}(|x|+\alpha x)^{-\delta\left(\mu+\frac{1}{n p}\right)-1} d x \int_{\mathbf{R} \backslash[-1,1]}(|y|+\beta y)^{-\left(\mu-\frac{1}{n q}\right)-1} d y \\
= & K_{\beta}\left(\sigma+\frac{1}{n q}\right)\left[(1-\alpha)^{\frac{-\delta}{n}-1}+(1+\alpha)^{\frac{-\delta}{n}-1}\right] n-O(1) .
\end{aligned}
$$

If there exists a constant $k \leq K(\sigma)$ such that (15) is valid when replacing $K(\sigma)$ by $k$, then in particular, we have

$$
\frac{1}{n} \tilde{I}=\frac{1}{n} \int_{-\infty}^{\infty} \int_{-\infty}^{\infty} \frac{\tilde{f}(x) \tilde{g}(y)}{\left[1+(|x|+\alpha x)^{\delta}(|y|+\beta y)\right]^{\lambda}} d y d x<\frac{k}{n} \tilde{L}
$$

In view of the above results, it follows that

$$
\begin{aligned}
& K_{\beta}\left(\sigma+\frac{1}{n q}\right)\left[(1-\alpha)^{\frac{-\delta}{n}-1}+(1+\alpha)^{\frac{-\delta}{n}-1}\right]-\frac{1}{n} O \\
& \quad<k\left[(1-\alpha)^{-\frac{\delta}{n}-1}+(1+\alpha)^{-\frac{\delta}{n}-1}\right]^{\frac{1}{p}}\left[(1-\beta)^{\frac{1}{n}-1}+(1+\beta)^{\frac{1}{n}-1}\right]^{\frac{1}{q}} .
\end{aligned}
$$

For $n \rightarrow \infty$, we find

$$
\frac{4 B(\mu, \sigma)}{\left(1-\alpha^{2}\right)\left(1-\beta^{2}\right)} \leq 2 k\left(\frac{1}{1-\alpha^{2}}\right)^{\frac{1}{p}}\left(\frac{1}{1-\beta^{2}}\right)^{\frac{1}{q}},
$$

namely $K(\sigma) \leq k$. Hence, $k=K(\sigma)$ is the best possible constant factor of (15).

The constant factor $K(\sigma)$ in (12) is also the best possible. Otherwise, we can conclude a contradiction by (18) that the constant factor in (17) is not the best possible.

The theorem is proved.

Theorem 3 With regards to the assumptions of Theorem 2, replacing $p>1$ by $0<p<1$, we have the equivalent reverses of (12) and (15) with the best possible constant factor $K(\sigma)$.

Proof We only prove that the constant factor $K(\sigma)$ in the reverse of (15) is the best possible, and omit the others. If there exists a constant $k \geq K(\sigma)$ such that the reverse of (15) is valid when replacing $K(\sigma)$ by $k$, then in particular, for $n \in \mathbf{N}=\{1,2, \ldots\}, n>\frac{1}{|q| \sigma}$, we have

$$
\begin{aligned}
k & {\left[(1-\alpha)^{-\frac{\delta}{n}-1}+(1+\alpha)^{-\frac{\delta}{n}-1}\right]^{\frac{1}{p}}\left[(1-\beta)^{\frac{1}{n}-1}+(1+\beta)^{\frac{1}{n}-1}\right]^{\frac{1}{q}} } \\
& =\frac{k}{n} \tilde{L}<\frac{1}{n} \tilde{I}=\frac{1}{n} \int_{-\infty}^{\infty} \int_{-\infty}^{\infty} \frac{\tilde{f}(x) \tilde{g}(y)}{\left[1+(|x|+\alpha x)^{\delta}(|y|+\beta y)\right]^{\lambda}} d y d x \\
& \leq \frac{1}{n} \int_{E_{\delta}}(|x|+\alpha x)^{\frac{-\delta}{n}-1} \varpi_{\delta}\left(\sigma+\frac{1}{n q}, x\right) d x \\
& =K_{\beta}\left(\sigma+\frac{1}{n q}\right)\left[(1-\alpha)^{\frac{-\delta}{n}-1}+(1+\alpha)^{\frac{-\delta}{n}-1}\right] .
\end{aligned}
$$


For $n \rightarrow \infty$, we obtain that $k \leq K(\sigma)$. Hence, $k=K(\sigma)$ is the best possible constant factor of the reverse of (15).

The theorem is proved.

\section{Operator expressions and a remark}

For $p>1, \frac{1}{p}+\frac{1}{q}=1, \delta \in\{-1,1\},-1<\alpha, \beta<1, \mu, \sigma>0, \mu+\sigma=\lambda$, we set the following functions: $\varphi(x):=(|x|+\alpha x)^{p(1-\delta \sigma)-1}, \psi(y):=(|y|+\beta y)^{q(1-\sigma)-1}$, where from

$$
\psi^{1-p}(y)=(|y|+\beta y)^{p \sigma-1} \quad(x, y \in \mathbf{R}) .
$$

Define the following real normed linear spaces:

$$
\begin{aligned}
& L_{p, \varphi}(\mathbf{R}):=\left\{f ;\|f\|_{p, \varphi}:=\left(\int_{-\infty}^{\infty} \varphi(x)|f(x)|^{p} d x\right)^{\frac{1}{p}}<\infty\right\}, \\
& L_{p, \psi^{1-p}}(\mathbf{R}):=\left\{h ;\|h\|_{p, \psi^{1-p}}:=\left(\int_{-\infty}^{\infty} \psi^{1-p}(y)|h(y)|^{p} d y\right)^{\frac{1}{p}}<\infty\right\}, \\
& L_{q, \psi}(\mathbf{R}):=\left\{g ;\|g\|_{q, \psi}:=\left(\int_{-\infty}^{\infty} \psi(y)|g(y)|^{q} d y\right)^{\frac{1}{q}}<\infty\right\} .
\end{aligned}
$$

In view of Theorem 1 , for any $f \in L_{p, \varphi}(\mathbf{R})$, we set

$$
h(y):=\int_{-\infty}^{\infty} \frac{1}{\left[1+(|x|+\alpha x)^{\delta}(|y|+\beta y)\right]^{\lambda}} f(x) d x \quad(y \in \mathbf{R}) .
$$

By (12) we have

$$
\|h\|_{p, \psi^{1-p}}=\left(\int_{-\infty}^{\infty} \psi^{1-p}(y)|h(y)|^{p} d y\right)^{\frac{1}{p}} \leq K(\sigma)\|f\|_{p, \varphi}<\infty .
$$

Definition 2 Define an extended Hilbert's integral operator in the whole plane

$$
T: L_{p, \varphi}(\mathbf{R}) \rightarrow L_{p, \psi^{1-p}}(\mathbf{R})
$$

as follows: For any $f \in L_{p, \varphi}(\mathbf{R})$, there exists $T f=h \in L_{p, \psi^{1-p}}(\mathbf{R})$.

In view of (20), the operator $T$ is bounded with

$$
\|T\|=\sup _{f(\neq \theta) \in L_{p, \varphi}(\mathbf{R})} \frac{\|T f\|_{p, \psi^{1-p}}}{\|f\|_{p, \varphi}} \leq K(\sigma) .
$$

Since by Theorem 2 the constant factor in (20) is the best possible, we have

$$
\|T\|=K(\sigma)=\frac{2 B(\mu, \sigma)}{\left(1-\beta^{2}\right)^{1 / p}\left(1-\alpha^{2}\right)^{1 / q}} .
$$

If we define the normal inner product of $T f$ and $g$ as follows:

$$
(T f, g):=\int_{-\infty}^{\infty}\left\{\int_{-\infty}^{\infty} \frac{1}{\left[1+(|x|+\alpha x)^{\delta}(|y|+\beta y)\right]^{\lambda}} f(x) d x\right\} g(y) d y
$$


then we can rewrite (15) and (12) as the following equivalent operator expressions:

$$
(T f, g)<\|T\| \cdot\|f\|_{p, \varphi}\|g\|_{q, \psi}, \quad\|T f\|_{p, \psi} 1-p<\|T\| \cdot\|f\|_{p, \varphi} .
$$

Remark 1 (i) In Theorem 2 , for $\delta=-1$, replacing $(|x|+\alpha x)^{\lambda} f(x)$ by $f(x)$, we have

$$
0<\int_{-\infty}^{\infty}(|x|+\alpha x)^{p(1-\mu)-1} f^{p}(x) d x<\infty
$$

and the following equivalent inequalities with the homogeneous kernel and the best possible constant factor $K(\sigma)=\frac{2 B(\mu, \sigma)}{\left(1-\beta^{2}\right)^{1 / p}\left(1-\alpha^{2}\right)^{1 / q}}$ :

$$
\begin{aligned}
& \left\{\int_{-\infty}^{\infty}(|y|+\beta y)^{p \sigma-1}\left[\int_{-\infty}^{\infty} \frac{f(x)}{(|x|+\alpha x+|y|+\beta y)^{\lambda}} d x\right]^{p} d y\right\}^{\frac{1}{p}} \\
& <\frac{2 B(\mu, \sigma)}{\left(1-\beta^{2}\right)^{1 / p}\left(1-\alpha^{2}\right)^{1 / q}}\left[\int_{-\infty}^{\infty}(|x|+\alpha x)^{p(1-\mu)-1} f^{p}(x) d x\right]^{\frac{1}{p}}, \\
& \int_{-\infty}^{\infty} \int_{-\infty}^{\infty} \frac{f(x) g(y)}{(|x|+\alpha x+|y|+\beta y)^{\lambda}} d x d y \\
& <\frac{2 B(\mu, \sigma)}{\left(1-\beta^{2}\right)^{1 / p}\left(1-\alpha^{2}\right)^{1 / q}\left[\int_{-\infty}^{\infty}(|x|+\alpha x)^{p(1-\mu)-1} f^{p}(x) d x\right]^{\frac{1}{p}}} \\
& \quad \times\left[\int_{-\infty}^{\infty}(|y|+\beta y)^{q(1-\sigma)-1} g^{q}(y) d y\right]^{\frac{1}{q}} .
\end{aligned}
$$

(ii) For $\alpha=\beta=0$, inequality (24) reduces to (6), and (17) reduces to

$$
\begin{aligned}
& \int_{-\infty}^{\infty} \int_{-\infty}^{\infty} \frac{f(x) g(y)}{(1+|x y|)^{\lambda}} d x d y \\
& \quad<2 B(\mu, \sigma)\left[\int_{-\infty}^{\infty}|x|^{p(1-\sigma)-1} f^{p}(x) d x\right]^{\frac{1}{p}}\left[\int_{-\infty}^{\infty}|y|^{q(1-\sigma)-1} g^{q}(y) d y\right]^{\frac{1}{q}} .
\end{aligned}
$$

(iii) For $p=q=2, \mu=\sigma=\frac{\lambda}{2}, f(-x)=f(x), g(-y)=g(y)(x, y>0)$, inequality (6) reduces to (4). Hence, inequality (6) is an extended Hilbert's integral inequality in the whole plane, and inequality (15) is a more general form of (6) with parameters.

\section{Conclusions}

In this paper, by introducing independent parameters and interval variables, applying the weight functions and the technique of real analysis, an extended Hilbert's integral inequality in the whole plane with parameters and a best possible constant factor is provided in Theorem 2. The equivalent forms, the reverses, and the related homogeneous forms with particular parameters are considered. An extended Hilbert's integral operator in the whole plane is defined, and the operator expressions for the equivalent inequalities are obtained. The method of weight functions is very important, which helps us to prove the equivalent inequalities with the best possible constant factor. The lemmas and theorems provide an extensive account of this type of inequalities. 


\section{Funding}

This work is supported by the National Natural Science Foundation (No. 61772140) and Science and Technology Planning Project of Guangzhou City (No. 201707010229). We are grateful for this help.

\section{Competing interests}

The authors declare that they have no competing interests.

\section{Authors' contributions}

BY carried out the mathematical studies, participated in the sequence alignment, and drafted the manuscript. LH and YL participated in the design of the study and performed the numerical analysis. All authors read and approved the final manuscript.

\section{Author details}

${ }^{1}$ College of Mathematics and Statistics, Jishou University, Jishou, P.R. China. ${ }^{2}$ Department of Mathematics, Guangdong University of Education, Guangzhou, P.R. China.

\section{Publisher's Note}

Springer Nature remains neutral with regard to jurisdictional claims in published maps and institutional affiliations.

Received: 31 March 2018 Accepted: 7 August 2018 Published online: 20 August 2018

\section{References}

1. Hardy, G.H., Littlewood, J.E. Pólya, G.: Inequalities. Cambridge University Press, Cambridge (1934)

2. Hardy, G.H.: Note on a theorem of Hilbert concerning series of positive terms. Proc. Lond. Math. Soc. 23(2), Records of Proc. xlv-xlvi (1925)

3. Mitrinović, D.S., Pečarić, J.E., Fink, A.M.: Inequalities Involving Functions and Their Integrals and Derivatives. Kluwer Academic, Boston (1991)

4. Yang, B.C.: On Hilbert's integral inequality. J. Math. Anal. Appl. 220, 778-785 (1998)

5. Wang, D.X., Guo, D.R.: Introduction to Especial Functions. Science Press, Beijin (1979)

6. Li, Y.J., He, B.: On inequalities of Hilbert's type. Bull. Aust. Math. Soc. 76(1), 1-13 (2007)

7. Yang, B.C.: The Norm of Operator and Hilbert-Type Inequalities. Science Press, Beijing (2009)

8. Xin, D.M., Yang, B.C.: A Hilbert-type integral inequality in the whole plane with the homogeneous kernel of degree -2. J. Inequal. Appl. 2011, Article ID 401428 (2011)

9. Wang, A.Z., Yang, B.C.: A new Hilbert-type integral inequality in the whole plane with the non-homogeneous kernel. J. Inequal. Appl. 2011, 123 (2011)

10. Xie, Z.T., Zeng, Z.: A new Hilbert-type inequality in whole plane with the homogeneous kernel of degree 0. i-manag. J. Math. 2(1), 13-19 (2013)

11. Rassias, M., Yang, B.C.: A Hilbert-type integral inequality in the whole plane related to the hyper geometric function and the beta function. J. Math. Anal. Appl. 428(2), 1286-1308 (2015)

12. Yang, B.C.: On the norm of an integral operator and application. J. Math. Anal. Appl. 321, 182-192 (2006)

13. Yang, B.C.: On the norm of a Hilbert's type linear operator and applications. J. Math. Anal. Appl. 325, 529-541 (2007)

14. Arpad, B., Choonghong, O.: Best constant for certain multi linear integral operator. J. Inequal. Appl. 2006, 28582 (2006)

15. Kuang, J.C.: Applied Inequalities. Shangdong Science Technic Press, Jinan (2004)

16. Kuang, J.C.: Real and Functional Analysis (Continuation) (Second Volume). Higher Education Press, Beijing (2015)

\section{Submit your manuscript to a SpringerOpen ${ }^{\circ}$ journal and benefit from:}

- Convenient online submission

- Rigorous peer review

- Open access: articles freely available online

- High visibility within the field

- Retaining the copyright to your article

Submit your next manuscript at $\boldsymbol{~ s p r i n g e r o p e n . c o m ~}$ 